\title{
Are there Upper Cretaceous sedimentary rocks preserved on Sørkapp Land, Svalbard?
}

\author{
Morten Smelror ${ }^{1} \&$ Geir Birger Larssen ${ }^{2}$ \\ ${ }^{1}$ Geological Survey of Norway (NGU), 7491 Trondheim, Norway. \\ ${ }^{2}$ Lundin Norway AS, P.O. Box 794, 9488 Harstad, Norway. \\ E-mail corresponding author (Morten Smelror): morten.smelror@ngu.no
}

Paleogene formations on eastern Sørkapp Land contain common, reworked, Middle and Late Cretaceous terrestrial and marine microfloras. This observation questions the Late Cretaceous age reported for the Firkanten Formation in the Øyrlandet Graben on western Sørkapp Land, as the pollen and spores reported from this graben may not be in situ. The present palynostratigraphic data from the eastern Sørkapp Land suggest that the Firkanten and Basilika formations were deposited in a relatively short time-period during the Early to Mid Thanetian. The youngest Cretaceous deposits documented from the Carolinefjellet Formation in eastern Sørkapp Land are of Late Albian age.

Keywords: Paleogene, Cretaceous, stratigraphy, dinoflagellates, Sørkapp, Svalbard, Arctic

Received 16. November 2015 / Accepted 30. May 2016 / Published online 10. August 2016

\section{Introduction}

The ages and chronology of the Cenozoic formations on Svalbard have been a ticklish problem for decades (see reviews in Manum \& Throndsen, 1986 and Čepek \& Krutszh, 2001). Some of the initial studies based on land plants and molluscs suffered from the low stratigraphic resolution of this material. Likewise, the very abundant terrestrial palynomorph assemblages found in these deltaic to shallow-marine sequences of the Cenozoic succession generally only contain long-ranging species of limited value for high-resolution biostratigraphy (Manum, 1962; Cepek \& Krutszh, 2001). More precise age determinations have been obtained from foraminifera, nannofossils and dinoflagellate cysts (Manum, 1960; Feyling-Hansen \& Ulleberg, 1984; Head, 1984; Manum \& Throndsen, 1986; Matthiessen, 1986; Smelror, 1996; Čepek, 2001; Harding et al., 2011) and from geochemical data (Dypvik et al., 2011; Harding et al., 2011), but these fossils and geochemical records are confined to intervals of strong marine influence.

The present study was carried out on behalf of the Norwegian Petroleum Directorate (NPD) with the objective to investigate the potential for more precise biostratigraphic dating of Paleogene rocks from outcrop sections on eastern Sørkapp Land (Fig. 1). The main goal was to constrain the ages of the youngest Cretaceous and the Paleogene lithostratigraphic units mapped in the area. In addition to the in situ microfloras, which document a late Early to early Late Paleocene age for the Firkanten and Basilika formations on eastern Sørkapp Land, the examined samples also contain common reworked Aptian-Albian and Late Cretaceous pollen, spores and dinoflagellate cysts. This observation apparently questions the Late Cretaceous age reported for the Firkanten Formation in the Øyrlandet Graben on western Sørkapp Land by Krutzsch (2001). 


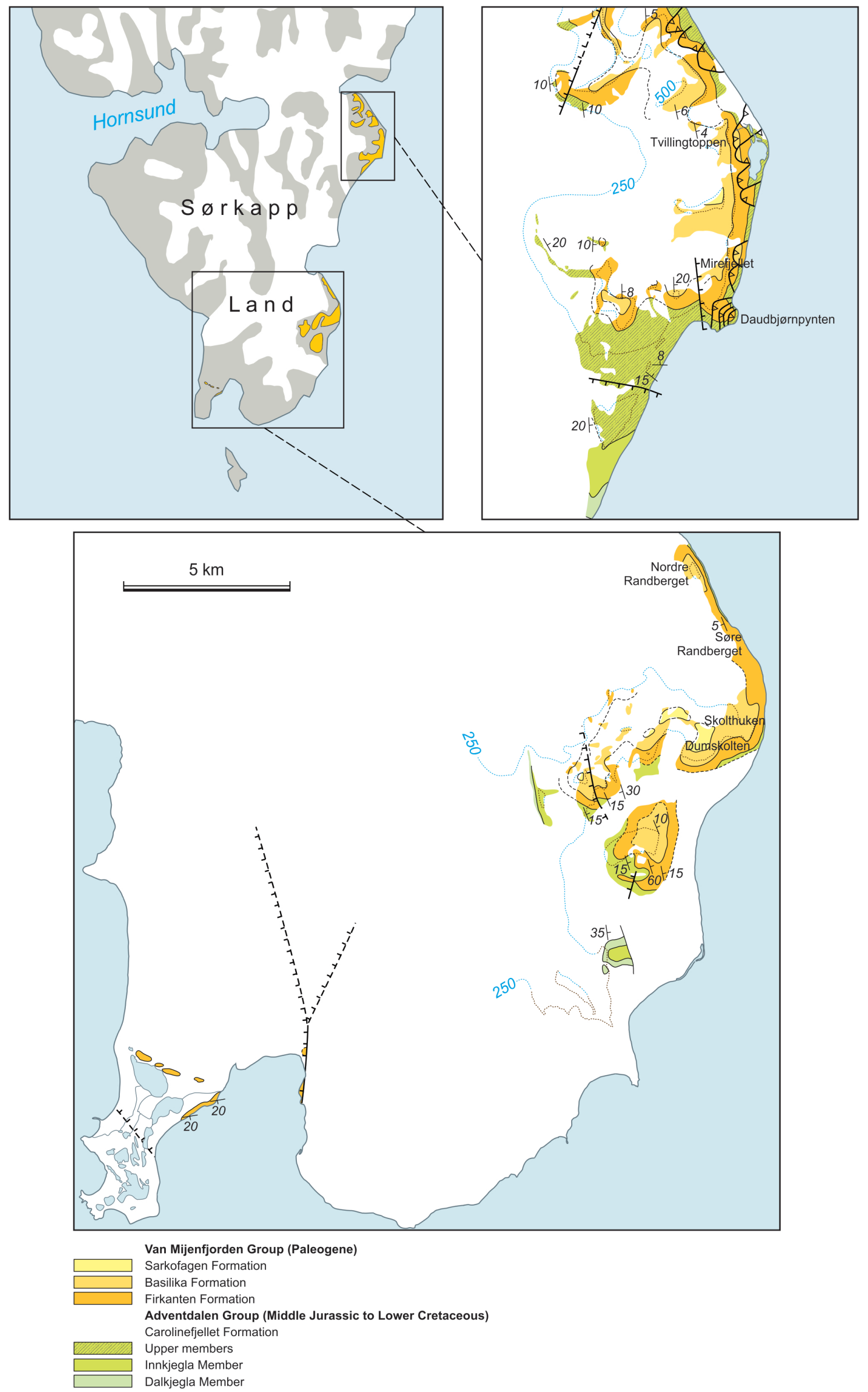

Figure 1. Outcrop of Tertiary formations on Sørkapp Land, Svalbard (based on the maps of Dallmann (1991) and Winsnes et al. (1993). 


\section{The Cretaceous-Tertiary boundary strata on Svalbard}

On Svalbard, the boundary between the Cretaceous and the Tertiary sedimentary successions is marked by a major hiatus corresponding to the Late Cretaceous and earliest Tertiary time interval. The hiatus is well documented in the Central Spitsbergen Basin and on Sørkapp Land (Fig. 2). Further, a similar hiatus is well documented from exploration wells and seismic correlations over large areas on the western Barents Shelf.

The youngest confirmed Cretaceous rocks on Svalbard are Aptian-Albian shale, siltstone and sandstone of the Carolinefjellet Formation (Fig. 2; Parker, 1967;

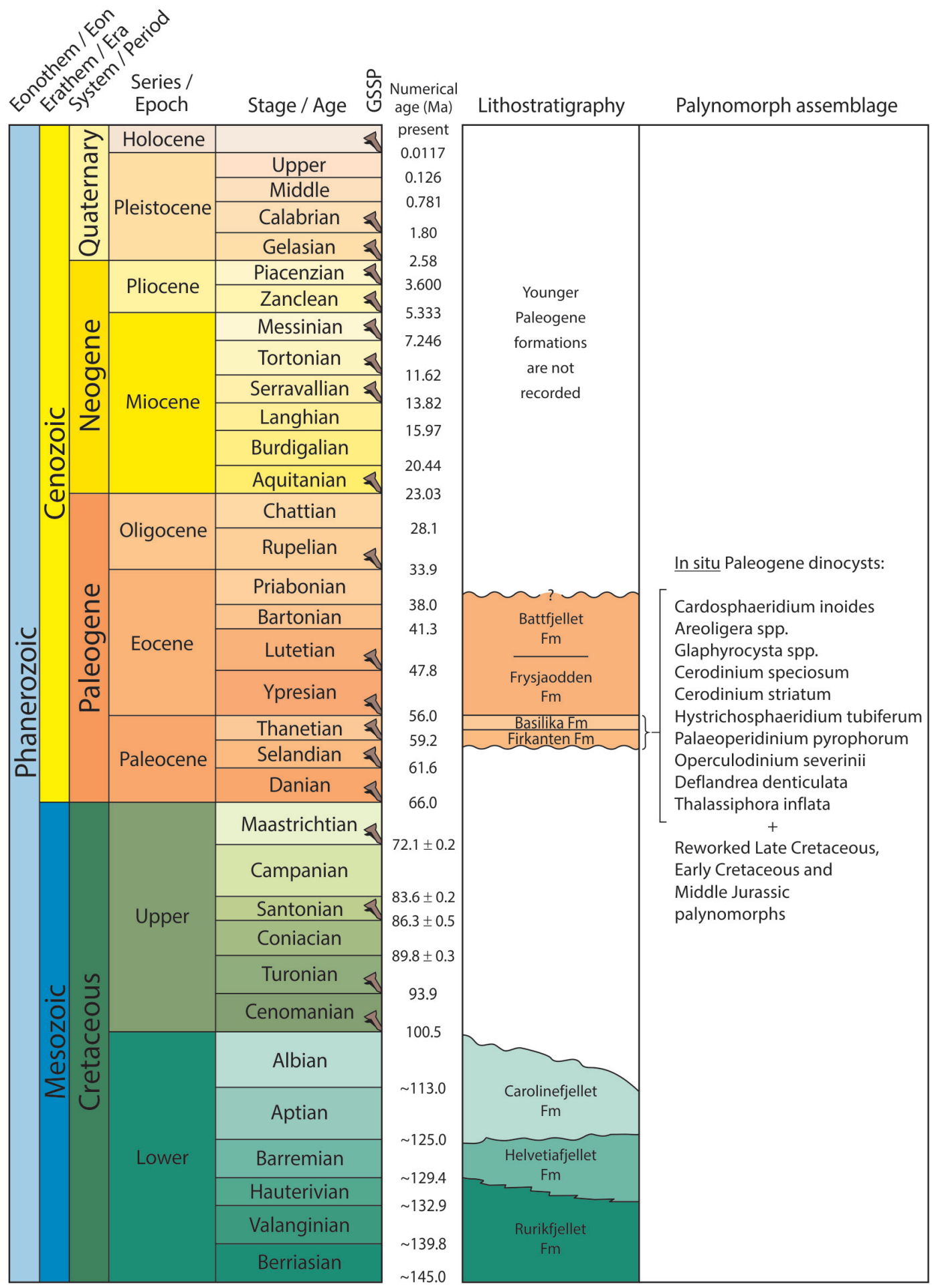

Figure 2. Cretaceous and Paleogene chronostratigraphy and lithostratigraphy of Svalbard, with a list of selected in situ and dinoflagellate cysts recovered from Paleogene formations on Sørkapp Land. 


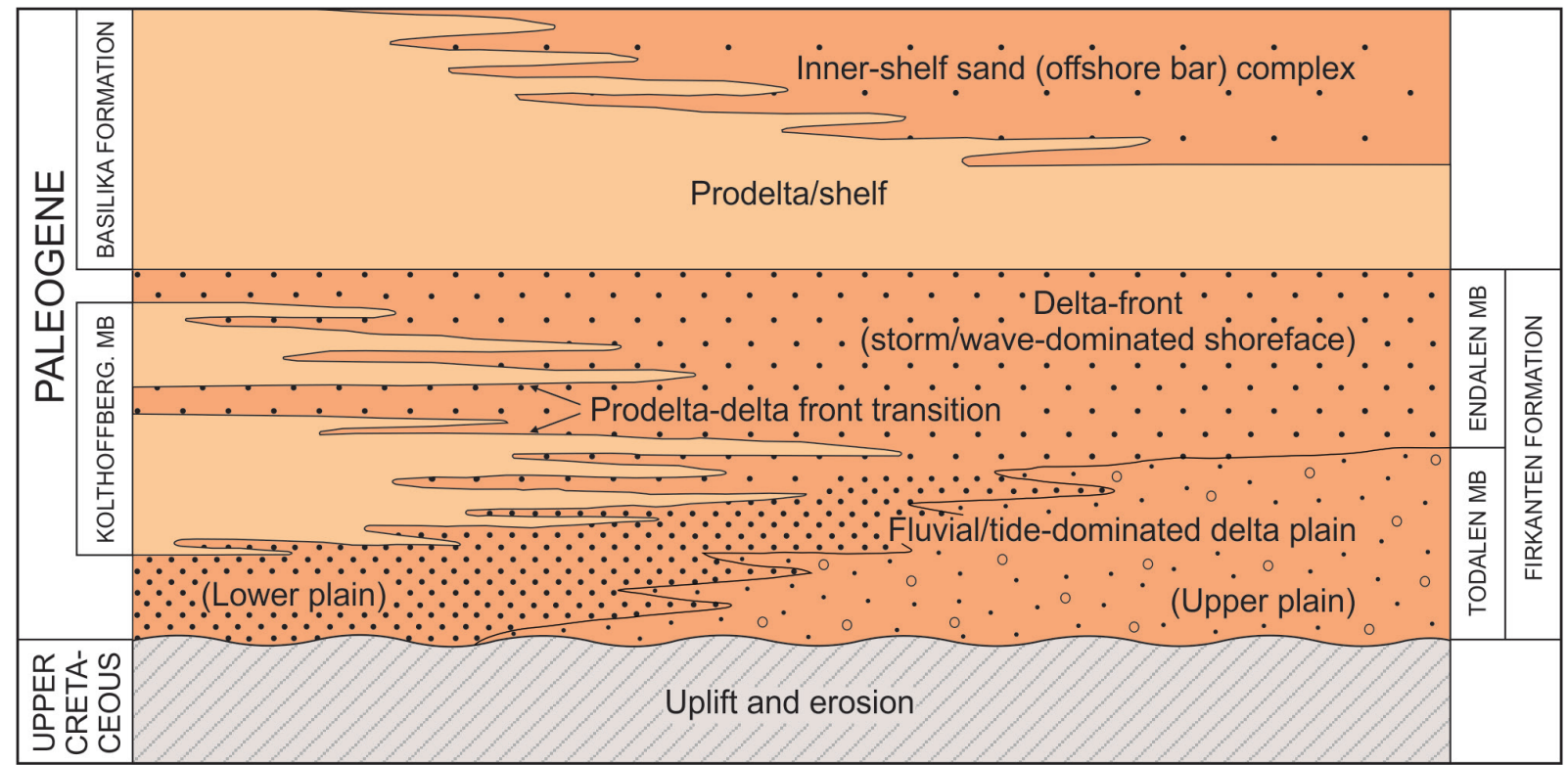

Figure 3. Outline stratigraphy and depositional conditions of the lower and middle parts of the Paleogene succession along a southwestnortheast transect crossing the Central Basin of Spitsbergen (based on Nagy, 2005).

Nagy, 1970; Dallmann, 1999). The formation is divided into four members. The youngest member is the Schönrockfjellet Member, dated as Albian by ammonites (Nagy, 1970). The member represents marine, moderately deep shelf environments, and it is locally preserved in Heer Land on Spitsbergen (Dallmann, 1999).

Overlying the Early Cretaceous deposits on Spitsbergen is the Paleocene-?Oligocene Van Mijenfjorden Group (Figs. 2, 3; Dallmann, 1999). The oldest sedimentary rocks of this group constitute the Paleocene Firkanten Formation, a unit of conglomerates, sandstone, shale and coal deposited in alluvial/deltaic to shallow-marine environments. The lowermost part of the Firkanten Formation comprises the Grønfjorden Bed, a basal conglomerate overlying the Carolinefjellet Formation with a low-angle unconformity (Dallmann, 1999).

The Firkanten Formation thickens and deepens from north-northeast towards south and west (HellandHansen, 1990; Dallmann et al., 1993). Towards the south in the Central Tertiary Basin, the lowermost Firkanten Formation is represented by sandstone, shale and coals of the Todalen Member (Dallmann, 1999). The lower, coalbearing Todalen Member of the formation represents a stacked series of deltaic lobes, while the upper part assigned to the Endalen Member shows a transgression repeated shoaling-upward barrier shoreline deposits building repeatedly from the northeast (Helland-Hansen, 1990). Overlying the basal Grønfjorden Bed and the Todalen Member are the Endalen Member and the Kolthoffberget Member. The latter member represents repeated shoaling-upward settings in a prodelta or shallow shelf, whereas the Endalen represents a deltaic or barrier shoreline deposit building repeatedly from the northeast (Helland-Hansen, 1990; Dallmann, 1999).

The Basilika Formation comprises shale, siltstone and mudstone deposited in a shelf setting. The lower boundary of the formation is well defined by the incoming of thick shales over the sandstones of the underlying Firkanten Formation (Dallmann, 1999). The formation is dated as Late Paleocene based on palynology (Manum \& Throndsen, 1986) and foraminifera (Nagy et al., 2000; Nagy, 2005).

\section{Material and methods}

A total of 53 samples from different locations on eastern Sørkapp Land were analysed for their palynological content. The samples were taken from locations at Daudbjørnpynten, Skolthuken, Mirefjellet, Randberget, Dumskolten and east of Tvillingtoppen (Fig. 1). More information on the locations of the examined sections and the outcrop of the Tertiary formation on eastern Sørkapp Land can be found on the geological map of Sørkapp in 1:100,000 scale (Winsnes et al., 1993). All samples were prepared following standard palynological procedures at the NPD laboratory. The examined palynological slides are stored at the IKU Petroleum Research core-store in Trondheim. With few exceptions the analysed samples yielded determinable palynomorphs. The preservation of the material and the productivity of the samples, however, were generally modest to rather poor. 


\section{Age of the Firkanten and Basilika formations}

The samples from the Firkanten and Basilika formations on Sørkapp Land contain dinoflagellate cyst assemblages of late Early to early Late Paleocene age (Figs. 4-12). Most samples contain common to abundant Paleoperidinium pyrophorum, and common Areoligerea/Glaphyrocysta spp., together with Cerodinium speciosum, Cerodinium striatum and Hystrichosphaeridium tubiferum. One sample from Randberget $(19.0 \mathrm{~m})$ contains common Operculodinium severinii (Fig. 7). Rare specimens of Alisocysta spp. were found at the Dumskolten II section (340.0 m; Fig. 9), while Deflandrea denticulate and Thalassiphora inflata were found in the Dumskolten III section (Fig. 10, at $619.0 \mathrm{~m}$ and $631.0 \mathrm{~m}$, respectively).

The consistent occurrences of Cerodinium speciosum and Cerodinium striatum appear to provide good evidence of a late Early to early Late Paleocene age for the deposits. According to Powell (1992), C. striatum ranges from the late Early to early Late Paleocene in the North Sea area (i.e., in beds time-equivalent to the NP4 to NP7 calcareous nannoplankton zones).

Nøhr-Hansen \& Sheldon (2000) reported C. striatum from Danian deposits in the Nuussuaq Basin in West Greenland, while Nøhr-Hansen (2012) reported the youngest occurrence of Cerodinium striatum and the Acme of Areoligera spp. in the Late Selandian Klitterhorn Member (upper Sediment Bjerge Formation) in the Kangerlussuaq Basin, southern East Greenland. Nagy et al. (2004) recovered C. striatum in Selandian to Ypresian deposits (i.e., strata correlated to the BSP2BSP4 Foraminiferal zones) of the Torsk Formation in the southwestern Barents Sea.

According to the stratigraphic range published by Powell (1992), the presence of C. speciosum indicates an early Late Paleocene age (Selandian-Early Thanetian) corresponding to the NP5-NP7 zones for the Basilika and Firkanten formations on eastern Sørkapp Land.

An age no younger than Early Thanetian (NP7) is also supported by the consistent occurrence of Palaeoperidinium pyrophorum. Mudge \& Bujak (2001) found that the youngest occurrences of common and abundant Palaeoperidinium pyrophorum correspond to the lower NP6 zone in the Faeroe-Shetland Basin. However, more sporadic occurrences of this species are reported from younger Early Paleogene strata (NP9-

Figure 4. Range chart of dinoflagellate cysts recovered from the Daudbjørnpynten section, Sørkapp Land. Abbreviations: Alb. Albian; Lt. Pal.- Late Paleocene; Car. - Carolinefjellet Formation; Firkant. - Firkanten Formation.

\begin{tabular}{|c|c|c|c|c|}
\hline \multirow{2}{*}{\begin{tabular}{|l|l|} 
Alb. \\
$\vec{\partial}$ \\
$\dot{\partial}$ & $\dot{y}$ \\
\end{tabular}} & \multicolumn{3}{|c|}{ Lt. Pal. } & \multirow{2}{*}{$\mid \begin{array}{l}\text { Age } \\
\text { Depth } \\
\mathrm{m} \quad\end{array}$} \\
\hline & & 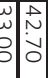 & & \\
\hline & & & $>$ & Cyclopsiella spp \\
\hline & & & & Exochosphaeridium sp. \\
\hline & & & & cf. Microdinium sp. \\
\hline$\times \mid>$ & $\times$ & $x$ & $\times$ & Spiniferites ramosus \\
\hline & & & $x^{x}$ & Cerodinium speciosum \\
\hline & & & $\times$ & Diconodinium spp. \\
\hline & & & $\times$ & Florentinia ferox \\
\hline & & & $\times$ & Palaeocystodinium golzowense \\
\hline & & & $\times$ & Wallodinium sp. \\
\hline & & & $\times$ & aff. Raphidodinium sp. \\
\hline & & $\times$ & $\times \mid$ & ?Areoligera/Glaphorocysta spp. \\
\hline & & $\times$ & $\times$ & Fromea fragilis \\
\hline & & $\times$ & $\times$ & Hystrochosphaeridium tubiferum \\
\hline & & $\times$ & $\times$ & Laciniadinium spp \\
\hline & & $\times$ & $\times \mid$ & Palaeoperidinium pyrophorum \\
\hline & & $\times$ & $\times$ & Thalassiphora cf. delicata \\
\hline$\times$ & & $\times$ & $\times$ & Cleistosphaeridium spp. \\
\hline$\times$ & & & $\times$ & Odontochitina operculata \\
\hline$\times$ & & & $\times$ & Oligosphaeridium pulcherrimum \\
\hline$\times$ & $\times$ & $\times$ & $\times$ & Oligosphaeridium complex \\
\hline$\times$ & & $\times$ & & Cordosphaeridium inodes \\
\hline & & $\times$ & & Lejeunecysta hyalina \\
\hline & & $\times$ & & Lithodinia sp. \\
\hline & & $\times$ & & Subtilisphaera sp. \\
\hline & & $\times$ & & ? Hapsocysta sp. \\
\hline & & $\times$ & & Pterodinium cingulatum \\
\hline$\times$ & & $x$ & & cf. Palaeoperidinium pyrophorum \\
\hline & $\times$ & & & cf. Leberidocysta sp. \\
\hline & $\times$ & & & Leptodinium sp. \\
\hline & $\times$ & & & Canninga spp \\
\hline & $\times$ & & & Cribroperidinium cf. orthoceras \\
\hline & $\times$ & & & Exochosphaeridium bifidum \\
\hline & $\times$ & & & Gonyalulacysta sp. \\
\hline & $\times$ & & & Hapsocysta? benteae \\
\hline & $\times$ & & & Litosphaeridium siphonophorum \\
\hline & $\times$ & & & Palaeoglenodinium cretaceum \\
\hline & $\times$ & & & Vesperopsis sp. \\
\hline$|\times|>$ & $\times$ & & & Cribroperidinium sp. \\
\hline$\times>$ & $\times$ & & & Ellipsoidictyum imperfectum \\
\hline$\times \mid>$ & $\times$ & & & Vesperopsis mayi \\
\hline$|\times|>$ & & & & Hystrichosphaeridium sp. \\
\hline$\times \mid>$ & $\times$ & & & Canningia sp. A \\
\hline$\times$ & & & & Circulodinium distinctum \\
\hline$\times$ & & & & Circulodinium spp. \\
\hline$\times$ & & & & Exochospheardium phragmites \\
\hline$\times$ & & & & Fromea cf. expolita \\
\hline$\times$ & & & & Kleithriasphaeridium sp. \\
\hline$\times$ & & & & Palaeoperidinium cretaceum \\
\hline$\times$ & & & & Spinidinium spp. \\
\hline |Car & & Firkar & & Lithostratigraphic unit \\
\hline
\end{tabular}




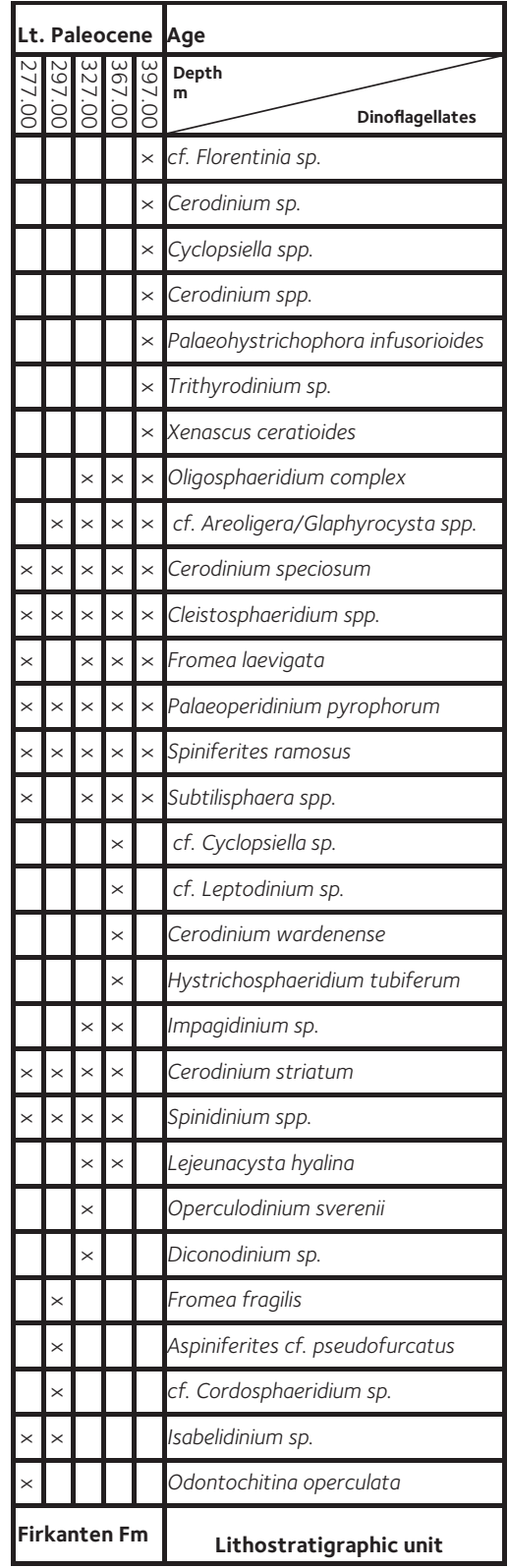

Figure 5. Range chart of dinoflagellate cysts recovered from the Skolthuken section, Sørkapp Land.

\begin{tabular}{|c|c|c|c|c|c|c|c|}
\hline \multicolumn{7}{|c|}{ Late Paleocene } & |Age \\
\hline$\vec{N}$ & & & & & 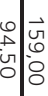 & ने & Dinoflagellates \\
\hline & & & & & & $\times$ & $\times$ Cerodinium sp. A \\
\hline & & & & $\times$ & & & Florentina feroks \\
\hline & & & $\times$ & & & $\times$ & Ceratiopsis sp. 1 Malm et al., 1984 \\
\hline & & & $\times x$ & $\times$ & & $|\times| \times$ & $\times$ Odontochitina operculata \\
\hline & & $x$ & & & & $\times$ & $\times$ Impagidinium spp. \\
\hline & $x$ & & $\times x$ & $\times$ & $x$ & $\times$ & $\times$ Cerodinium spp. \\
\hline & $\times$ & $\times$ & & $\times$ & & $\times$ & $\times$ Spinidinium spp. \\
\hline$\times$ & $x$ & & $\times>$ & $\times \mid \times$ & $\times \mid \times$ & $\times \mid \times$ & $\times$ Cerodinium speciosum \\
\hline$\times$ & $\times$ & & & & $x$ & $\times \mid \times$ & $\times$ Lejeunacysta hyalina \\
\hline$\times$ & $\times$ & $\times x$ & $\times \mid \times$ & $\times \mid \times$ & $\times \mid \times$ & $\times$ & $\times$ Oligosphaeridium complex \\
\hline$\times$ & $\times$ & $\times>$ & $\times>$ & $\times \times$ & $\times \times$ & $\times \mid \times$ & $\times$ Phalaeoperidinium pyrophorum \\
\hline$\times$ & $\times$ & $\times \mid \times$ & $\times \mid \times$ & $\times \mid \times$ & $\times$ & $|\times| \times$ & $\times$ Spiniferites ramosus \\
\hline & & & & & & $\times$ & Cleistophaeridium sp. \\
\hline$\times$ & $\times$ & $\times>$ & $\times$ & & $\times$ & $\times$ & Subtilisphaera spp. \\
\hline & & & & & $\times$ & & Pterodinium cingulatum \\
\hline & & & & $\times$ & $\times$ & & Laciniadinium spp. \\
\hline & & $\times \mid x$ & $\times x$ & $\times$ & $x$ & & Chlamydophorella nyei \\
\hline & & & & & $\times$ & & Muderongia tetracantha \\
\hline & $\times$ & $\times>$ & $\times \times$ & $\times \times$ & $\times$ & & Cleistosphaeridium ssp. \\
\hline$\times$ & $\times$ & $\times>$ & $\times>$ & $\times \times$ & $\times$ & & Cerodinium striatum \\
\hline & & & & $x$ & & & ?Hapsocysta sp. \\
\hline & & & & $\times$ & & & Exochosphaeridium sp. \\
\hline & & & & $\times$ & & & Fromea fragilis \\
\hline & & & & $\times$ & & & Palaeohystrichophora infusoriooides \\
\hline & & & $\times>$ & $\times$ & & & Impletosphaeridium spp. \\
\hline & & $\times$ & & $\times$ & & & Isabelidinium acuminatum \\
\hline & $\times$ & & & $\times$ & & & Exochosphaeridium bifidum \\
\hline & & $\times \mid \times$ & $\times$ & & & & Chatangiella spp. \\
\hline$\times$ & $\times$ & $x \mid \times$ & $\times$ & & & & ?Aeroligera/Glaphyrocysta spp. \\
\hline$\times$ & $\times$ & & $\times$ & & & & Hystrichosphaeridium tubiferum \\
\hline & & $\times$ & & & & & Cometodium sp. \\
\hline & & $x$ & & & & & Evansia evittii \\
\hline & & $\times$ & & & & & Exochosphaeridium phragmites \\
\hline & & $\times$ & & & & & Fromea laevigata \\
\hline & & $x$ & & & & & Gonyaukacysta helicoidea \\
\hline & & $\times$ & & & & & Substilisphaera perlucida \\
\hline & & $\times$ & & & & & Systematophora sp. \\
\hline & & $\times$ & & & & & Thalassiphora pelagica \\
\hline & & $\times$ & & & & & Trithyrodinium sp. \\
\hline & $\times \mid$ & $\times$ & & & & & Cordosphaeridium inodes \\
\hline & $\times$ & & & & & & Achilleodinium cf. biformoldes \\
\hline & $x$ & & & & & & Spiniferites cornutus \\
\hline$\times$ & $x$ & & & & & & Circulodinium distinctum \\
\hline$\times$ & & & & & & & Oligosphaeridium pulcerrimum \\
\hline$\times$ & & & & & & & Operculodinum severinii \\
\hline Fir & rkan & hte & & & م? & & Lithostratigraphic unit \\
\hline
\end{tabular}

Figure 6. Range chart of dinoflagellate cysts recovered from the Mirefjellet section, Sørkapp Land. Abbreviation: B. - Basilika Formation. 


\begin{tabular}{|c|c|c|}
\hline \multicolumn{2}{|c|}{ Lt. Pal. } & \\
\hline 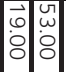 & & $\begin{array}{l}\text { Depth } \\
\mathrm{m}\end{array}$ \\
\hline & $\times$ & Chatangiella sp. \\
\hline & $\times$ & Florentina sp. \\
\hline & $\times$ & Impletosphaeridium spp. \\
\hline$x$ & $\times$ & Cordospaheridium inodes \\
\hline$\times$ & $\times$ & Odontochitina operculata \\
\hline$\times$ & $|\times|$ & Palaeocystodinium sp. \\
\hline$\times$ & $\times$ & ?Aeroligera/Glaphyrocysta spp. \\
\hline$\times \mid \times$ & $\times$ & Cerodinium speciosum \\
\hline$\times|\times|$ & $\times$ & Cleistophaeridium spp. \\
\hline$|\times| \times \mid$ & $\times$ & Oligosphaeridium conplex \\
\hline$\times \times$ & $\times$ & Palaeoperidinium pyrophorum \\
\hline$\times \times$ & $\times$ & Spinidinum spp. \\
\hline$\times$ & & Spiniferites ramosus \\
\hline$\times$ & & Ceratiopsis sp. 1 Malm er al., 1984 \\
\hline$\times$ & & Florentina mantelii \\
\hline$\times$ & & Fromea laevigata \\
\hline$\times$ & & Palaeophystrichophora infusorioides \\
\hline$\times$ & & Substilisphaera sp. \\
\hline$\times \times$ & & Hystrichsphaeridium tubiferum \\
\hline$\times$ & & Operculodinium severinii \\
\hline rin & & Lithos \\
\hline
\end{tabular}

Figure 7. Range chart of dinoflagellate cysts recovered from the Randberget section, Sørkapp Land. Abbreviations: Lt. Pal. - Late Paleocene; Firk. - Firkanten Formation.

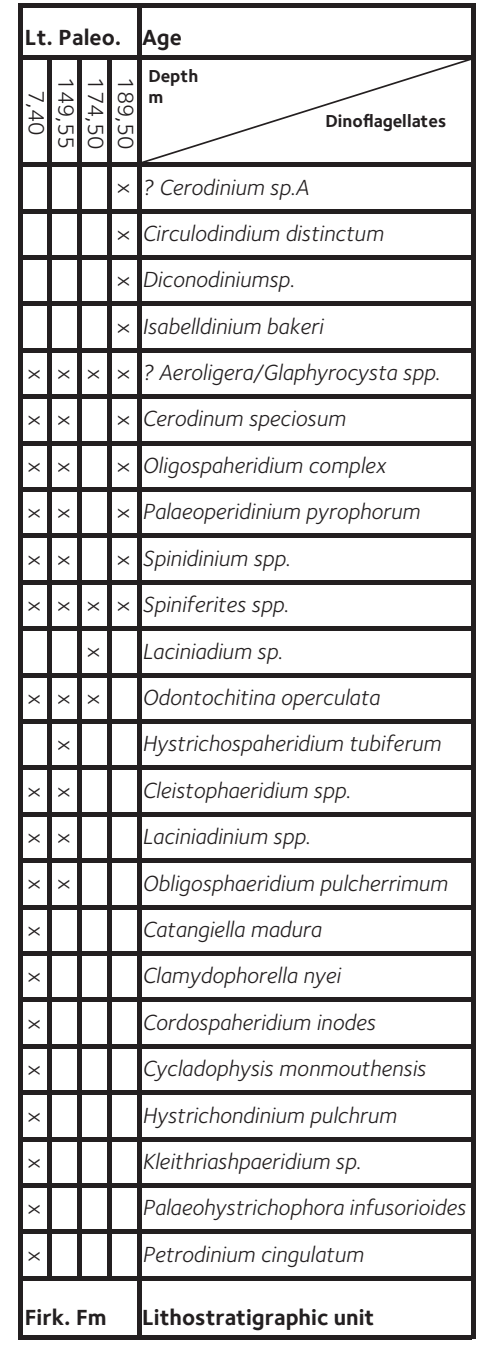

Figure 8. Range chart of dinoflagellate cysts recovered from the Dumskolten I section, Sørkapp Land. Abbreviations: Lt. Paleo.- Late Paleocene; Firk. Fm. - Firkanten Formation.

published by Powell (1992) are based on North Sea data, and should not be expected to be completely concurrent with the high latitude ranges. However, the age evidence seems consistent enough to conclude that the Firkanten and Basilika formations on Sørkapp Land are of Early to Mid Thanetian age.

\section{Age of the uppermost Carolinefjellet Formation}

Samples from the uppermost Carolinefjellet Formation were collected at Daudbjørnpynten (2 samples, Fig. 4) and at the section east of Tvillingtoppen (2 samples, Fig. 11).

At Daudbjørnpynten, the lowest sample from the Carolinefjellet Formation $(1.0 \mathrm{~m})$ yielded a moderately diverse dinoflagellate assemblage, characterised by 


\begin{tabular}{|c|c|c|c|c|}
\hline \multicolumn{4}{|c|}{ Lt. Paleocene } & \multirow{2}{*}{$\begin{array}{ll} \\
\text { Depth } \\
\mathrm{m}\end{array}$} \\
\hline & 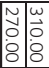 & 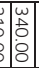 & 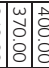 & \\
\hline & & & $\times$ & Spinidinum balmei \\
\hline & & & $\times \times$ & Oligospaheridium anthophorum \\
\hline & & $\times$ & $\times \mid \times$ & Cyclonephelium membraniphorum \\
\hline & $\times$ & $\times$ & $\times$ & Hystrichodinium pulchrum \\
\hline & $\times$ & $\times$ & $\times \mid \times$ & Spinidinium spp. \\
\hline & $\times x$ & $\times$ & $\times$ & Hystricosphaeridium tubiferum \\
\hline & $x$ & & $\times$ & Implestosphaeridium whitei \\
\hline & $x \times$ & 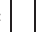 & $|x| \times$ & Palaeocystodinium golzowense \\
\hline & $\times x$ & $\times$ & $\times x$ & Spiniferites cf. pseudofurcatus \\
\hline$x$ & $\times x$ & $\times$ & $\times x$ & ?Aeroligera/Glaphyrocysta spp. \\
\hline$\times$ & $\times x$ & $\times$ & $x \mid \times$ & Chlamydophorella nyei \\
\hline$x$ & $\times x$ & $\times$ & $\times x$ & Circulondinium distinctum \\
\hline$x$ & $\times \mid x$ & $\times$ & $|\times| \times$ & Cleistospaheridium armatum \\
\hline$\times x$ & $\times x$ & $\times$ & $\times \times$ & Cleistospaheridium ssp. \\
\hline$\times$ & $\times$ & $\times$ & $\times$ & Exochosphaeridium phragmites \\
\hline$\times$ & $\times \mid x$ & $\times$ & $\times \mid \times$ & Laciniadinium spp. \\
\hline$x$ & $\times x$ & $\times$ & $\times x$ & Odontochitina operculata \\
\hline$\times$ & $\times x$ & $\times$ & $\times \mid \times$ & Oligospaheridium complex \\
\hline$x$ & $\times x$ & $\times$ & $\times \times$ & Palaeohystrichophora infusoriodes \\
\hline$\times$ & $\times x$ & $\times$ & $|\times| \times$ & Palaeroperidinium pyrophorum \\
\hline | & $\times \mid \times$ & $\times$ & $\times \mid \times$ & Spiniferites ramsus \\
\hline$x$ & & & $\times$ & Surculosphaeridium? longifurcatum \\
\hline & & & $\times$ & Cibroperidinium edwardsii \\
\hline & & & $\times$ & Elytrocysta drugii \\
\hline & $\times$ & $\times$ & $\times$ & Ceratiopsis sp. 1 Malm et al. 1984 \\
\hline$\times$ & $\times$ & . & $\times$ & ?Florentinia sp. \\
\hline$\times$ & $\times$ & $\times$ & $\times$ & Isabelidinium acumlnatum \\
\hline \multirow[t]{10}{*}{$\times$} & $\times x$ & 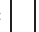 & $\times$ & Trichodinium castanea \\
\hline & & $\times$ & & ?Alisocysta sp. \\
\hline & & $\times$ & & Cycladophysis monmouthensis \\
\hline & & $\times$ & & Hystrichodinium vogtil \\
\hline & & $\times$ & & Hystrichosphaeropsis sp. \\
\hline & & $\times$ & & Kleithriasphaeridium sp. \\
\hline & & $\times$ & & Odontochitina costata \\
\hline & & $\times$ & & Rottenesita borussica \\
\hline & $x$ & $\times$ & & Cerodinium speciosum \\
\hline & $\times$ & $\times$ & & Phelodinium magnificum \\
\hline$\times$ & & $\times$ & & inopterygium sp. \\
\hline$x$ & $\times$ & $\times$ & & Exochosphaeridium bifidum \\
\hline$\times$ & & $\times$ & & Palaeogelenodinium cretaceum \\
\hline & $x$ & & & ?Apteodinium sp. \\
\hline & $x$ & 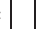 & & ?Disphaera sp. \\
\hline & $\times$ & 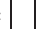 & & Trithyrodinium sp. \\
\hline & $x$ & 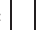 & & Exhospaeridium sp. \\
\hline & $x$ & . & & Fromea laevigata \\
\hline & $x$ & 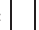 & & Impletsophaeridium sp. \\
\hline & $x$ & . & & Isabelidinium bakeri \\
\hline & $x$ & 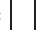 & & Petrodinium sp. \\
\hline & $x$ & 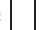 & & Spongodinium delitiense \\
\hline & $\times \mid x$ & . & & Impagidinium spp. \\
\hline & $\times x$ & 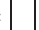 & & Palaeoperidinum cretaceum \\
\hline$x$ & $\times x$ & 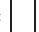 & & Pterodinium cingulatum \\
\hline$x$ & $x \times$ & 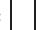 & & Substilisphaera spp. \\
\hline & $\times$ & & & Florentinia mantellii \\
\hline$x$ & $\times$ & & & Trithyrodinum fragile \\
\hline$\times$ & $\times$ & & & Chatangiella spectabilis \\
\hline$x \times$ & & & & Cyclopsiella spp. \\
\hline$\times$ & & & & ?Hapsocysta sp. \\
\hline$\times$ & & & & Cerodinium striatum \\
\hline$\times$ & & & & Cibroperidinium sp. \\
\hline$x$ & & & & Oligosphaeridium pulcherrimum \\
\hline$\times$ & & & & Trithyrodinium suspectum \\
\hline$x$ & & & & Xenascus ceratioldes \\
\hline & Lithostratigraphic unit \\
\hline
\end{tabular}

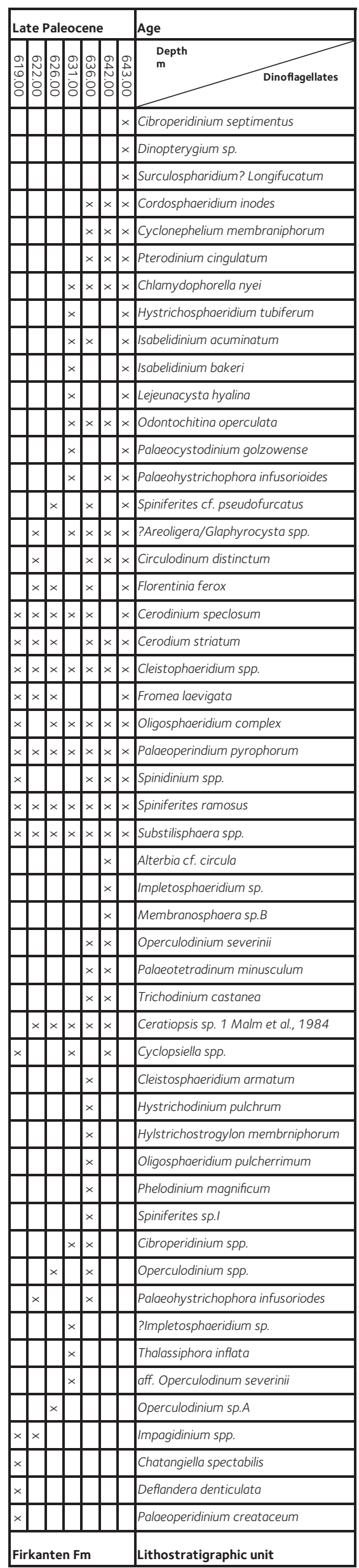

Figure 10.

Range chart of dinoflagellate cysts recovered from the Dumskolten III section, Sørkapp Land. 


\begin{tabular}{|c|c|c|c|}
\hline \multicolumn{2}{|c|}{ Albian } & L P. & \multirow{2}{*}{$\begin{array}{ll}\text { Age } \\
\text { Depth } \\
\mathrm{m}\end{array}$} \\
\hline & & & \\
\hline & & $x$ & cf. Oligosphaeridium sp. \\
\hline & & $\times$ & $\times$ cf. Palaeoperidinium pyrophorum \\
\hline & & $\times \mid \times$ & Spiniferites spp. \\
\hline & & $\times \mid \times$ & Cerodinium spp. \\
\hline & & $\times$ & Cerodinium speciosum \\
\hline & & $\times$ & Cerodinium striatum \\
\hline & & $\times$ & Circulodinium spp. \\
\hline & & $\times$ & Fromea laevigata \\
\hline & & $\times$ & Subtilisphaera spp. \\
\hline & & $\times$ & Thalassiphora pelagica \\
\hline & $\times$ & $\times$ & Hystrichosphaeridium tubiferum \\
\hline & $\times$ & $x$ & Paleoeperidinium pyrophorum \\
\hline & $\times \mid \times$ & $x$ & Cleistosphaeridium spp. \\
\hline$\times$ & $\times \mid \times$ & $\times$ & Oligosphaeridium complex \\
\hline$\times$ & & $\times$ & Oligosphaeridium pulcherrimum \\
\hline$\times$ & $\times \mid \times$ & $x$ & Spiniferites ramosus \\
\hline & $\times$ & & Cribroperidinium sp. \\
\hline & $\times$ & & cf. Trithyrodinium sp. \\
\hline$\times$ & $\times$ & & Odontochitina operculata \\
\hline$\times$ & $\times$ & & Palaeoperidinium cretaceum \\
\hline$\times$ & & & Canningia spp. \\
\hline$\times$ & & & Hystrichosphaeridium spp. \\
\hline$\times$ & & & Impagidinium sp. \\
\hline$\times$ & & & Vesperopsis mayi \\
\hline & & Firk. & Lithostratigraphic unit \\
\hline
\end{tabular}

Figure 11. Range chart of dinoflagellate cysts recovered from the east of Tvillingtoppen section, Sørkapp Land. Abbreviations: L P. - Late Paleocene; Carol. - Carolinefjellet Formation; Firk. - Firkanten Formation.

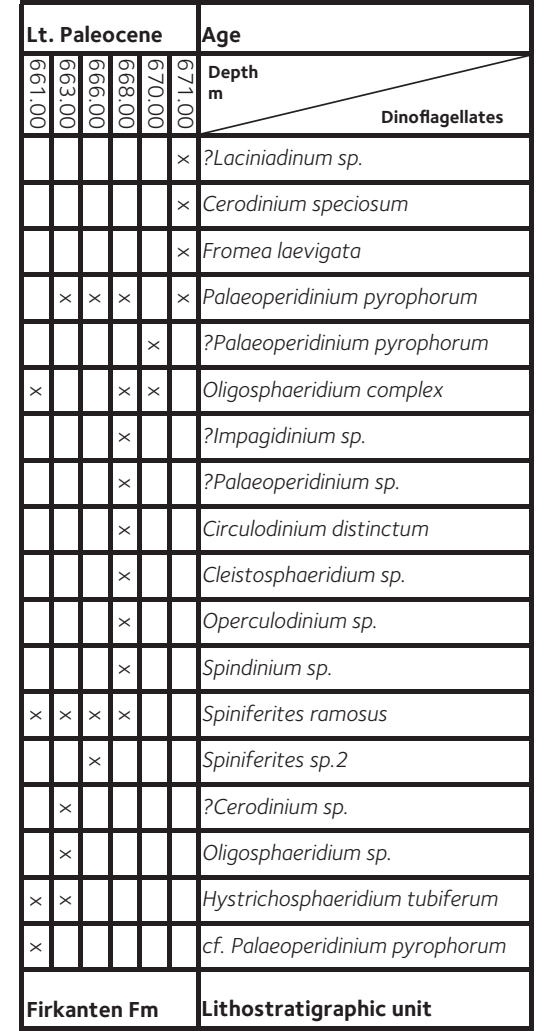

Figure 12. Range chart of dinoflagellate cysts recovered from the Dumskolten East section, Sørkapp Land. dominant Oligosphaeridium complex and common Cleistosphaeridium spp. and Spiniferites ramosus (Fig. 4). The presence of Ellipsoidictyum imperfectum suggests a general Late Aptian to early Late Albian age. The upper sample $(29.0 \mathrm{~m})$ also contained common $O$. complex, together with $E$. imperfectum. The sample further contained Hapsocysta? benteae which, according to NøhrHansen (1993), is a good marker for the Albian in East Greenland. Another species, Vesperopsis mayi, found in the assemblage is a typical Aptian to middle Late Albian species. The record of Litosphaeridium siphonophorum further restricts the age of the sample to the Late Albian, as this species has previously not been reported from pre-Late Albian strata (Nøhr-Hansen, 1993).

The lower sample $(40.0 \mathrm{~m})$ from the east of Tvillingtoppen section contained a low diversity dinoflagellate cyst assemblage, with the most prominent species being Oligosphaeridium complex (Fig. 11). The occurrence of Vesperopsis mayei in the sample is evidence for a general Apian to middle Late Albian age. The upper sample proved less productive and contained no agediagnostic species, with the exception of Operculodinium operculata which is consistent with a Mid Cretaceous age.

In conclusion, the combined dinoflagellate cyst assemblages recovered from the uppermost Carolinefjellet Formation in eastern Sørkapp Land point to a Late Albian age for the youngest Cretaceous strata in the area.

\section{Reworked palynomorph assemblages in the Tertiary formations}

Reworked Cretaceous dinoflagellates and terrestrial playnomprphs are common to abundant in samples through all the investigated outcrop sections.

The samples from the Firkanten Formation (5 samples from the Kolthoffberget Member and 2 samples from the 
Endalen Member) and the two samples from the Basilika Formation at the Mirefjellet section all contain reworked Cretaceous dinoflagellate cysts (Fig. 6). Most of these are long-ranging species, but the presence of Subtilisphaera perlucida and Gonyaulacysta helicoidea at $26.0 \mathrm{~m}$, and Trithyrodinium sp. and Isabelidinium acuminatum at $26.0 \mathrm{~m}$ and $63.5 \mathrm{~m}$, respectively, shows that both Lower and Upper Cretaceous sediments have been reworked into the Kalthoffberget Member. A single specimen of Evansia evittii further documents reworking from Middle Jurassic strata. Further upwards, the presence of Muderongia tectracantha at $95.5 \mathrm{~m}$ is evidence for reworking from the Lower Cretaceous into the Endalen Member. Aquilapollenties found at $159.0 \mathrm{~m}$ may have been redeposited from Upper Cretaceous sediments, but this pollen may also occur in situ in the Paleocene.

In the Dumskolten I section the recovery of Chatangiella madura at $7.4 \mathrm{~m}$ is evidence of recycling from Upper Cretaceous sediments (Fig. 7). The record of taeniated pollen at $174.0 \mathrm{~m}$ points to reworking from PermoTriassic strata. In the Dumskolten II section (Fig. 9), characteristic reworked Late Cretaceous species include Spinidinium balmei $(400.0 \mathrm{~m})$, Elytrocysta druggii $(370.0 \mathrm{~m})$, Isabelidinium acuminatum $(240.0 \mathrm{~m}, 310.0$ $\mathrm{m}, 340.0 \mathrm{~m}, 370.0 \mathrm{~m})$, Isabelidinium bakeri $(310.0 \mathrm{~m})$, Spondinimium delitiense $(310.0 \mathrm{~m})$, Trithyrodinium fragile $(270.0 \mathrm{~m})$, Trithyrodinium suspectum $(240.0 \mathrm{~m})$, Palaeoglenodinium cretaceous $(240.0,340.0 \mathrm{~m})$, and Xenascus ceratoides. The Late Cretaceous-Paleocene Aquilapollenties was recovered at $270.0 \mathrm{~m}$ and $370.0 \mathrm{~m}$. The reworked Cretaceous palynomorphs found in the Dumskolten III section have also been sourced from both Lower and Upper Cretaceous strata. The Late Cretaceous species include Isabelininium acuminatum $(631.0 \mathrm{~m}, 636.0 \mathrm{~m}, 643.0 \mathrm{~m})$, Isabelidinium bakeri $(631.0$ $\mathrm{m}, 643.0 \mathrm{~m})$, Phelodinium magnificum $(636.0 \mathrm{~m})$ and Chatangiella spectabilis $(619.0 \mathrm{~m})$.

No age-diagnostic reworked Cretaceous palynomorphs have been found in the Firkanten Member at the Dumskolten east section (Fig. 12) and the east of Tvillingtoppen section (Fig. 11). Only Early Cretaceous reworked dinoflagellate cysts and terrestrial palynomorphs have been found in the 3 examined samples from the Todalen Member at the Daudbjørnpynten section. In the Skolthuken section (Fig. 4), however, both Early and Late Cretaceous reworked dinoflagellate cysts are found. Recycling from Upper Cretaceous sediments is shown by the presence of Isabelidinium sp. at $277.0 \mathrm{~m}$, and by Trithyrodinium sp. and Xenascus ceratoides at $397.0 \mathrm{~m}$.

\section{Discussion}

The present palynostratigraphic data from the eastern Sørkapp Land suggest that the Firkanten and Basilika formations were deposited in a relatively short timeperiod during the Early to Mid Thanetian. Further, calcareous nannofossils reported from the Firkanten Formation at Scott-Ruudfjellet in the northern part of Sørkapp Land by Čepek (2001) point to a Late Paleocene age.

Except for the report by Krutzsch (2001), no Upper Cretaceous sedimentary rocks have been documented from Svalbard, and consequently the report of an Upper Cretaceous microflora from Øyrlandet Graben on Sørkapp Land (Krutzsch, 2001) is intriguing. The Triprojectacites (Aquilapolles) microflora was found in one sample from undeformed, sandy sediments assigned to the Firkanten Formation by Dallmann et al. (1993).

Upper Cretaceous strata occur in NE Greenland (Balkwill et al., 1983; Håkansson et al., 1991) and they are also present in the Maud Basin and Tromsø Basin on the western Barents Shelf. While the Upper Cretaceous of NE Greenland contains terrestrial deposits (i.e., the Herlufsholm Strand Formation) and marine deposits (i.e., the Nakkehoved Formation), the Upper Cretaceous of the Hammerfest, Maud and Tromsø basins comprises marine claystones and siltstones deposited in an open marine environment (Faleide et al., 1993; Radmacher et al., 2013, 2014).

The present recovery of abundant Upper Cretaceous palynomorphs in eastern Sørkapp Land questions the assumption that the Upper Cretaceous microflora found in one single sample in the Øyrlandet Graben documents a Late Cretaceous age for this sample. On the other hand, the fact that no marine palynomorphs were reported in the sample from the Øyrlandet Graben may also suggest that this sample represents an isolated body of Upper Cretaceous terrestrial deposits. Thus, the question as to whether there are any Upper Cretaceous deposits preserved on Sørkapp Land still remain unsolved.

It must also be mentioned that Late Cretaceous dinoflagellate cysts, pollen and spores are commonly found reworked in the Paleogene and younger Cenozoic deposits in the western Barents Shelf basins (i.e., documented in a number of unpublished biostratigraphy well reports). Abundant reworked Late Cretaceous palynomorphs have also previously been reported in the Upper Pliocene-Pleistocene deposits recovered at ODP Sites 910 and 911 on the Yermak Plateau (Willard, 1996) and at Site 986 west of southern Svalbard (Smelror, 1999). As discussed by Willard (1996) and Smelror (1999), these documented findings are sourced from the Late Cretaceous Aquilapollenites (Triprojectacites) 
floral province. The subsequent records from Sørkapp Land (Krutzsch, 2001, and the present paper) support the assumption that the Western Barents Shelf (including Svalbard) was a part of this province in Late Cretaceous time.

\section{Conclusions}

The Paleogene formations on eastern Sørkapp Land contain common, reworked, Middle and Late Cretaceous terrestrial and marine microfloras (Figs. 4-12). The present palynostratigraphic data from eastern Sørkapp Land suggest that the Firkanten and Basilika formations were deposited in a relatively short time-period during the Early to Mid Thanetian. The youngest Cretaceous deposits documented from the Carolinefjellet Formation in eastern Sørkapp Land are of Late Albian age.

The observations of common, reworked, Upper Cretaceous palynomorphs in the Firkanten Formation question the Late Cretaceous age reported for the Firkanten Formation in the Øyrlandet Graben in western Sørkapp Land, as the pollen and spores reported from this graben may not be in situ. On the other hand, the fact that no marine palynomorphs were found in the sample from the Øyrlandet Graben may also suggest that this sample may represent an isolated body of Upper Cretaceous terrestrial deposits. Thus, the question as to whether there are Upper Cretaceous deposits preserved on Sørkapp Land still remains unsolved.

Acknowledgements. The present study is based on material sampled by the Norwegian Petroleum Directorate and petroleum companies from selected outcrops on Sørkapp Land, Svalbard, in 1995. Thanks are due to reviewers William Helland-Hansen and Henrik Nøhr-Hansen for helpful suggestions that led to improvements in the manuscript.

\section{References}

Balkwill, H.R., Cook, D.G., Dettermann, R.L., Embry, A.F., Håkansson, E., Miall, A.D., Poulton, T.P. \& Young, F.G. 1983: Arctic North America and northern Greenland. The Phanerozoic Geology of the World, II The Mesozoic B, 1-31.

Čepek, P. 2001: Paleogene Calcareous Nannofossils from the Firkanten and Sarsbukta Formations on Spitsbergen. Geologisches Jahrbuch B91, 535-547.

Čepek, P. \& Krutzsch, W. 2001: Conflicting Interpretations of the Tertiary Biostratigraphy of Spitsbergen and New Palynological results. Geologisches Jahrbuch B91, 551-599.

Dallmann, W.K. (ed.) 1999: Lithostratigraphic Lexicon of Svalbard. Norsk Polarinstitutt, Tromsø, $318 \mathrm{pp}$.

Dallmann, W.K., Andresen, A., Bergh, S.G., Maher, H.D. Jr. \& Ohta, Y. 1993: Tertiary fold-and-thrust belt of Spitsbergen, Svalbard. Norsk Polarinstitutt Meddelelser 128, 1-46.
Dypvik, H., Rieber, L., Burca, F., Rüther, D., Jargvoll, D., Nagy, J. \& Jochmann, M. 2011: The Paloecene-Eocene thermal maximum (PETM) in Svalbard - clay mineral and geochemical signals. Palaeogeography, Palaeoclimatology, Palaeoecology 302, 156-169.

Faleide, J.I., Vågnes, E. \& Gudlaugsson, S.T. 1993: Late Mesozoic-Cenozoic evolution of the south-western Barents Sea in a regional riftshear tectonic setting. Marine and Petroleum Geology 10, 186-214.

Feyling-Hansen, R. \& Ulleberg, K. 1984: A Tertiary-Quaternary section at Sarsbukta, Spitsbergen, Svalbard, and its foraminifera. Polar Research 2,77-106.

Harding, I.C., Charles, A.J., Marshall, J.E.A., Pälike, H., Roberts, P.A., Wilson, P.A., Jarvis, E., Thorne, R., Morris, E., Moremon, R., Pearce, R.B. \& Akbari, S. 2011: Sea level and salinity fluctuations during the Paleocene-Eocene Thermal Maximum in Arctic Spitsbergen. Earth and Planetary Science Letters 303, 97-107

Head, M. 1984: A palynological investigation of Tertiary strata at Renarodden, W. Spitsbergen. Abstract $6^{\text {th }}$ International Palynology Conference Calgary, 26 August to 1 September 1984, Calgary, Canada, p. 61.

Helland-Hansen, W. 1990: Sedimentation in a Paleogene foreland basin, Spitsbergen. American Association of Petroleum Geologists Bulletin 74, 260-272.

Håkansson, E., Heinberg, C. \& Stemmerik, L. 1991: Mesozoic and Cenozoic History of the Wandel Sea Basin Area. Bulletin Grønlands Geologiske Undersøgelse 160, 153-164.

Krutzsch, W. 2001: An Upper Cretaceous Microflora from Spitsbergen. Geologisches Jahrbuch B91, 693-713.

Manum, S.B. 1960: Some dinoflagellates and hystrichosphaerids from the lower Tertiary of Spitsbergen. Nytt Magsin for Botanikk 8, $17-18$.

Manum, S.B. 1962: Studies in the Tertiary flora of Spitsbergen, with notes on Tertiary floras of Ellesmere Island, Grrenland and Iceland. A palynological investigation. Norsk Polarinstutt Skrifter 125, 127 pp.

Manum, S.B. \& Throndsen, T. 1986: Age of Tertiary Formations on Svalbard. Polar Research 4, 103-131.

Matthiessen, J. 1986: Biostratigraphie tertiärer Ablagerubgen (Paläozän) am Van Keulenfjord (Spitsbergen) nach Dinoflagellaten Zysten. Diplom-Arbeit, University of Kiel, 94 pp.

Mudge, D.C. \& Bujak, J.P. 1996: Paleocene biostratigraphy and sequence stratigraphy of the UK central North Sea. Marine and Petroleum Geology 13, 295-312.

Mudge, D.C. \& Bujak, J.P. 2001: Biostratigraphic evidence for evolving palaeonvironments in the Lower Paleogene of the Faroe-Shetland Basin. Marine and Petroleum Geology 18, 577-590.

Nagy, J. 1970: Ammonite faunas and stratigraphy of Lower Cretaceous (Albian) rocks in southern Spitsbergen. Norsk Polarinstitutt Skrifter $152,1-58$.

Nagy, J. 2005: Delta-influenced foraminiferal facies and sequence stratigraphy of Paleocene deposits in Spitsbergen. Palaeogeography, Palaeoclimatology, Palaeoecology 222, 161-179.

Nagy, J., Kaminski, M.A., Kuhnt, W. \& Bremer, M. 2000: Agglutinated foraminifera from neritic to bathyal facies in the Paleogene of Spitsbergen and the Barents Sea. In Hart, M.B., Kaminski, M.A. \& Smart, C.W. (eds.): Proceedings of the Fifth International Workshop on Agglutinated Foraminifera, Grzybowski Foundation Special Publication 7, Grzybowski Foundation, Krakow, pp. 333-361.

Nagy, J., Kaminski, M.A., Gradstein, F. \& Johnson, K. 2004. Quantitative foraminiferal and palynomorph biostratigraphy of the Paleogene in the southwestern Barents Sea. In Bubik, M. \& Kaminski, M.A. (eds.): Proceedings of the Sixth International Workshop on Agglutinated Foraminifera, Grzybowski Foundation Special Publication 8, Grzybowski Foundation, Krakow, pp. 359-379.

Nøhr-Hansen, H. 1993: Dinoflagellate cyst stratigraphy of the Barremian to Albian, Lower Cretaceous, North-East Greenland. Bulletin Grønlands Geologiske Undersøgelse 166, 1-171.

Nøhr-Hansen, H. 2012: Palynostratigraphy of the Cretaceous-lower Palaeogene sedimentary succession in the Kangerlussuaq Basin, southern East Greenland. Review of Palaeobotany and Palynology 
178, 59-90.

Nøhr-Hansen, H. \& Sheldon, E. 2000: Palyno- and nannostratigraphic dating of the marine Paleocene succession in the Nuussuaq Basin, west Greenland. Geologiska Föreningens i Stockholm Förhandlingar 122, 115-116.

Nøhr-Hansen, H., Nielsen, L.H., Sheldon, E., Hovikoski, J. \& Alsen, P. 2011: Palaeogene deposits in North-East Greenland. Geological Survey of Denmark and Greenland Bulletin 23, 61-64.

Parker, J.R. 1967: The Jurassic and Cretaceous sequence in Spitsbergen. Geological Magazine 104, 487-505.

Powell, A.J. 1992: Dinoflagellate cysts of the Tertiary System. In Powell, A.J. (ed.): A stratigraphic Index of Dinoflagellate Cysts, British Micropaleontology Society Publications Series, Chapmann \& Hall, London, pp. 155-251.

Radmacher, W., Tyszka, J. \& Mangerud, G. 2013: Distribution and biostratigraphical significance of Heterosphaeridium bellii sp. nov. and other Late Cretaceous dinoflagellate cysts from the southwestern Barents Sea. Review of Palaeobotany and Palynology 201,29-40.

Radmacher, W., Tyszka, J., Mangerud, G. \& Pearce, M.A. 2014: Dinoflagellate cyst biostratigaphy of the Upper Albian to Lower Maastrichian in the southwestern Barents Sea. Marine and Petroleum Geology $57,109-121$.

Smelror, M. 1996: Biostratigraphy of the Paleogene formations on Sørkapp Land, Spitsbergen. IKU Report 25.2450.00/01/96, 38 pp.

Smelror, M. 1999: Pliocene-Pleistocene and redeposited dinoflagellate cysts from the western Svalbard margin (Site 986): Biostratigraphy, paleoenvironments, and sediment provenance. Proceedings of the Ocean Drilling Program, Scientific Results 162, 83-97.

Willard, D.A. 1996: Pliocene-Pleistocene pollen assemblages from the Yermak Plateau, Arctic Ocean: Sites 910 and 911. Proceedings of the Ocean Drilling Program, Scientific Results 151, 297-307.

Winsnes, T.S., Birkenmajer, K., Dallmann, W.K., Hjelle, A. \& Salvigsen, O. 1993: Geological Map Svalbard, C13G Sørkapp, Norsk Polarinstitutt Temakart Nr. 17, scale 1:100,000, Norsk Polarinstitutt. 